StaniseaW TrybuŁa (Wrocław)

\title{
MINIMAX MUTUAL PREDICTION OF MULTINOMIAL RANDOM VARIABLES
}

Abstract. The problem of minimax mutual prediction is considered for multinomial random variables with the loss function being a linear combination of quadratic losses connected with prediction of particular variables. The basic parameter of the minimax mutual predictor is determined by numerical solution of some equation.

1. Introduction. Suppose that $m$ statisticians take part in a prediction process, $m \geq 2$. Let a random variable $X_{i}=\left(X_{i 1}, \ldots, X_{i r}\right)$ be observed by the $i$ th statistician. The random variables $X_{i}, i=1, \ldots, m$, are independent and have the multinomial distribution with parameters $n_{i}$, $p=\left(p_{1}, \ldots, p_{r}\right)$. The statisticians do not know the observations of their partners but they know all the numbers $n_{i}$. They cooperate with each other. The problem solved in this paper is to determine the minimax mutual predictor

$$
d=\left[\begin{array}{cccc}
- & d_{12} & \ldots & d_{1 m} \\
d_{21} & - & \ldots & d_{2 m} \\
\ldots \ldots & \ldots & \ldots & \ldots . \\
d_{m 1} & d_{m 2} & \ldots & -
\end{array}\right]=:\left[d_{i j}\right]_{i, j=1}^{m},
$$

where $d_{i j}\left(X_{i}\right)=\left(d_{i j}^{(1)}\left(X_{i}\right), \ldots, d_{i j}^{(r)}\left(X_{i}\right)\right)$ is the predictor of $X_{j}=\left(X_{j 1}, \ldots\right.$ $\left.\ldots, X_{j r}\right)$ used by the $i$ th statistician to predict this random variable, $i, j=$ $1, \ldots, m, i \neq j$.

Thus each statistician observes only his "own" random variable and predicts only the others.

2000 Mathematics Subject Classification: Primary 62F15.

Key words and phrases: minimax mutual prediction, multinomial distribution, Bayes predictor. 
The total loss of all the statisticians is

$$
L(X, d)=\sum_{\substack{i, j=1 \\ i \neq j}}^{m} k_{i j} \sum_{l=1}^{r} c_{l}\left(d_{i j}^{(l)}\left(X_{i}\right)-X_{j l}\right)^{2}
$$

where $k_{i j} \geq 0, \sum_{i, j=1, i \neq j}^{m} k_{i j}>0, c_{l} \geq 0$ are constants. Without loss of generality we can assume that $c_{1} \geq \ldots \geq c_{r} \geq 0$.

Let $R(p, d)$ be the risk function connected with the predictor $d$,

$$
R(p, d)=E_{p}(L(X, d))=\sum_{\substack{i, j=1 \\ i \neq j}}^{m} k_{i j} \sum_{l=1}^{r} c_{l} E_{p}\left(d_{i j}^{(l)}\left(X_{i}\right)-X_{j l}\right)^{2} .
$$

We then look for a mutual predictor $d_{0}$ for which

$$
\sup _{p} R\left(p, d_{0}\right)=\inf _{d} \sup _{p} R(p, d) .
$$

2. Determining the minimax predictor in the main case. Let the random variables $X_{i}, i=1, \ldots, m$, be independent and distributed according to the multinomial law

$$
P_{p}\left(X_{i 1}=x_{i 1}, \ldots, X_{i r}=x_{i r}\right)=\frac{n_{i} !}{x_{i 1} ! \ldots x_{i r} !} p_{1}^{x_{i 1}} \ldots p_{r}^{x_{i r}} .
$$

Then the risk (2) takes the form

$$
R(p, d)=\sum_{\substack{i, j=1 \\ i \neq j}}^{m} k_{i j} \sum_{l=1}^{r} c_{l}\left[E_{p}\left(d_{i j}^{(l)}\left(X_{i}\right)-n_{j} p_{l}\right)^{2}+n_{j} p_{l}\left(1-p_{l}\right)\right] .
$$

Let us consider the predictors

$$
d_{i j}^{(l)}\left(X_{i}\right)=n_{j} \frac{X_{i l}+\alpha_{l}}{n_{i}+\gamma}, \quad i, j=1, \ldots, m, i \neq j, l=1, \ldots, r,
$$

where $\alpha_{l} \geq 0, \gamma>0$ and

$$
\sum_{l=1}^{r} \alpha_{l}=\gamma
$$

For this mutual predictor the risk is as follows:

$$
\begin{aligned}
& \sum_{\substack{i, j=1 \\
i \neq j}}^{R(p, d)} k_{i j} \sum_{l=1}^{r} c_{l}\left[E_{p}\left(n_{j} \frac{X_{i l}+\alpha_{l}}{n_{i}+\gamma}-n_{j} p_{l}\right)^{2}+n_{j} p_{l}\left(1-p_{l}\right)\right] \\
= & \sum_{\substack{i, j=1 \\
i \neq j}}^{m} k_{i j} \sum_{l=1}^{r} c_{l}\left\{\frac{n_{j}^{2}}{\left(n_{i}+\gamma\right)^{2}}\left[n_{i} p_{l}\left(1-p_{l}\right)+\left(\alpha_{l}-\gamma p_{l}\right)^{2}\right]+n_{j} p_{l}\left(1-p_{l}\right)\right\}
\end{aligned}
$$


Assume that

$$
\begin{aligned}
& =\sum_{\substack{i, j=1 \\
i \neq j}}^{m} k_{i j} \sum_{l=1}^{r} c_{l}\left\{\left[\frac{n_{j}^{2}}{\left(n_{i}+\gamma\right)^{2}}\left(-n_{i}+\gamma^{2}\right)-n_{j}\right] p_{l}^{2}\right. \\
& \left.+\left[\frac{n_{j}^{2}}{\left(n_{i}+\gamma\right)^{2}}\left(n_{i}-2 \alpha_{l} \gamma\right)+n_{j}\right] p_{l}+\frac{n_{j}^{2}}{\left(n_{i}+\gamma\right)^{2}} \alpha_{l}^{2}\right\} .
\end{aligned}
$$

$$
\varphi(\gamma):=\sum_{\substack{i, j=1 \\ i \neq j}}^{m} k_{i j}\left[\frac{n_{j}^{2}}{\left(n_{i}+\gamma\right)^{2}}\left(-n_{i}+\gamma^{2}\right)-n_{j}\right]=0
$$

Since $\varphi(0)<0$, the equation (6) always has a solution $\gamma>0$ if

$$
A=: \lim _{\gamma \rightarrow \infty} \varphi(\gamma)=\sum_{\substack{i, j=1 \\ i \neq j}}^{m} k_{i j} n_{j}\left(n_{j}-1\right)>0
$$

In this section we will suppose that the condition (7) holds and that $\gamma$ is a solution of (6).

Applying the formula (6) to (5) we obtain

$$
\begin{aligned}
R(p, d)= & \sum_{\substack{i, j=1 \\
i \neq j}}^{m} k_{i j} \frac{n_{j}^{2}}{\left(n_{i}+\gamma\right)^{2}} \sum_{l=1}^{r} c_{l}\left(\gamma^{2}-2 \gamma \alpha_{l}\right) p_{l} \\
& +\sum_{\substack{i, j=1 \\
i \neq j}}^{m} k_{i j} \frac{n_{j}^{2}}{\left(n_{i}+\gamma\right)^{2}} \sum_{l=1}^{r} c_{l} \alpha_{l}^{2} .
\end{aligned}
$$

Assume that $c_{2} \neq 0$. Let $l_{0}$ be the greatest index $l$ for which $c_{l} \neq 0$ and let

$$
L=\max _{s}\left\{s \leq l_{0}: \sum_{k=1}^{s} \frac{1}{c_{k}}>\frac{s-2}{c_{s}}\right\} .
$$

LEMMA. Under the above notation, for $l=L+1, \ldots, r$,

$$
q:=\frac{L-2}{\sum_{k=1}^{L} 1 / c_{k}} \geq c_{l} .
$$

Proof. Notice that the proof of the inequality (10) is only necessary for $l=L+1$. If $c_{L+1} \neq 0$ from (9) it follows that

$$
L-1 \geq c_{L+1} \sum_{k=1}^{L+1} \frac{1}{c_{k}}=1+c_{L+1} \sum_{k=1}^{L} \frac{1}{c_{k}} .
$$

The inequality (10) follows from (11).

If $c_{L+1}=0$ the inequality (10) obviously holds since $L \geq 2$. 
We shall prove the following theorem:

Theorem 1. If $A>0$ then the game defined by the statistical decision problem considered has a value and the mutual predictor $d=\left[d_{i j}\right]_{i, j=1}^{m}$ defined by (3) for

$$
\alpha_{l}= \begin{cases}\frac{\gamma}{2}\left(1-\frac{L-2}{c_{l} \sum_{k=1}^{L} 1 / c_{k}}\right) & \text { for } l=1, \ldots, L, \\ 0 & \text { for } l=L+1, \ldots, r,\end{cases}
$$

where $\gamma$ is the solution of (6), is minimax for the loss function given by (1).

Proof. Let the constants $\alpha_{l} \geq 0$ satisfy the equations

$$
\begin{array}{rlrl}
\gamma-2 \alpha_{l} & =\frac{a}{c_{l}} & \text { for } l & =1, \ldots, L, \\
\alpha_{l} & =0 & \text { for } l & l=L+1, \ldots, r .
\end{array}
$$

Taking into account the equations (4), (13) and (14) we obtain

$$
(L-2) \gamma=a \sum_{l=1}^{L} \frac{1}{c_{l}} .
$$

Since $\gamma$ and $L$ are known, the constant $a$ is known and $a \geq 0$ because $\gamma>0$ and $L \geq 2$. Moreover, from (10) and (15) it follows that

$$
a=q \gamma
$$

and from (13)-(15) we obtain the formula (12).

From (8) it follows that

$$
\begin{aligned}
& R(p, d) \\
& =\sum_{\substack{i, j=1 \\
i \neq j}}^{m} k_{i j} \frac{n_{j}^{2}}{\left(n_{i}+\gamma\right)^{2}}\left[\sum_{l=1}^{L} c_{l}\left(\gamma^{2}-2 \gamma \alpha_{l}\right) p_{l}+\sum_{l=L+1}^{r} c_{l} \gamma^{2} p_{l}+\sum_{l=1}^{r} c_{l} \alpha_{l}^{2}\right] \\
& \stackrel{(13)}{=} \sum_{\substack{i, j=1 \\
i \neq j}}^{m} k_{i j} \frac{n_{j}^{2}}{\left(n_{i}+\gamma\right)^{2}}\left[\sum_{l=1}^{L} a \gamma p_{l}+\sum_{l=L+1}^{r} c_{l} \gamma^{2} p_{l}+\sum_{l=1}^{L} c_{l} \alpha_{l}^{2}\right] \\
& \stackrel{(16)}{=} \sum_{\substack{i, j=1 \\
i \neq j}}^{m} k_{i j} \frac{n_{j}^{2}}{\left(n_{i}+\gamma\right)^{2}}\left[\sum_{l=1}^{L} q \gamma^{2} p_{l}+\sum_{l=L+1}^{r} c_{l} \gamma^{2} p_{l}+\sum_{l=1}^{L} c_{l} \alpha_{l}^{2}\right] .
\end{aligned}
$$

Thus $R(p, d)=$ const $=C$ if $\sum_{l=1}^{L} p_{l}=1$ and always, by the inequality (10), $R(p, d) \leq C$ for the mutual predictor $d$ defined by (3) where $\gamma$ and $\alpha_{l}$ are determined by (6) and (12). On the other hand, for any $d$ and the loss function (1) the Bayes risk $r(\pi, d)=E_{\pi}(R(p, d))$ attains its minimum if

$$
d_{i j}^{(l)}\left(X_{i}\right)=n_{j} E\left(p_{l} \mid X_{i}\right)
$$


In the above formulae $E_{\pi}(\cdot)$ denotes the expectation with respect to the prior distribution $\pi$ of the parameter $p=\left(p_{1}, \ldots, p_{r}\right)$ and $E\left(p_{l} \mid X_{i}\right)$ is the conditional expectation of $p_{l}$ for given $X_{i}$.

To prove that, assume that $p=\left(p_{1}, \ldots, p_{r}\right)$ is a random variable. Let $X_{i}=\left(X_{i 1}, \ldots, X_{i r}\right)$. The expression

$$
E_{\pi}\left(E_{p}\left(d_{i j}^{(l)}\left(X_{i}\right)-X_{j l}\right)^{2}\right)=E_{\pi}\left(E_{p}\left(d_{i j}^{(l)}\left(X_{i}\right)-n_{j} p_{l}\right)^{2}+n_{j} p_{l}\left(1-p_{l}\right)\right)
$$

attains its minimum when

$$
d_{i j}^{(l)}\left(X_{i}\right)=n_{j} E\left(p_{l} \mid X_{j}\right)=E\left(p_{l} \mid\left(X_{i 1}, \ldots, X_{i r}\right)\right) .
$$

Let the prior distribution $\pi$ of $p=\left(p_{1}, \ldots, p_{r}\right)$ be defined as follows:

$$
\begin{aligned}
& P\left(p_{1}+\ldots+p_{L}=1\right)=1, \quad p_{i} \geq 0, \\
& g\left(p_{1}, \ldots, p_{L}\right)=\frac{\Gamma(\gamma)}{\Gamma\left(\alpha_{1}\right) \ldots \Gamma\left(\alpha_{L}\right)} p_{1}^{\alpha_{1}-1} \ldots p_{L}^{\alpha_{L}-1},
\end{aligned}
$$

where $g$ is a density. For the prior density (18) and the loss function (1) the Bayes predictor is

$$
\begin{aligned}
d_{i j}^{(l)}\left(x_{i 1}, \ldots, x_{i L},\right. & 0, \ldots, 0) \\
& =n_{j} E\left(p_{l} \mid X_{i 1}=x_{i 1}, \ldots, X_{i L}=x_{i L}, X_{i l}=0 \text { for } l>L\right) \\
& = \begin{cases}n_{j} \frac{x_{i l}+\alpha_{l}}{n_{i}+\gamma} & \text { for } l=1, \ldots, L, \\
0 & \text { for } l=L+1, \ldots, r ; i, j=1, \ldots, m, i \neq j .\end{cases}
\end{aligned}
$$

Then $d=\left[d_{i j}\right]_{i, j=1}^{m}$ defined by (3) for $\gamma$ and $\alpha_{l}$ satisfying (6) and (12) is the Bayes predictor and from the Hodges-Lehmann theorem (see [2]) it follows that the game defined by the statistical decision problem considered has a value and this predictor is minimax.

3. Solution of the cases not solved in Section 2. Let $\varphi(\gamma)$ be defined by (6). Suppose that

$$
A=\lim _{\gamma \rightarrow \infty} \varphi(\gamma)=\sum_{\substack{i, j=1 \\ i \neq j}}^{m} k_{i j} n_{j}\left(n_{j}-1\right)=0 .
$$

Since $\varphi(0)<0$ and $\varphi(\gamma)$ is an increasing function of $\gamma$, in this case there does not exist any finite $\gamma>0$ for which $\varphi(\gamma)=0$. But it is easy to prove that in this case the minimax mutual predictor is obtained by taking into account the formula (12) and letting $\gamma \rightarrow \infty$ in (3),

$$
d_{i j}^{(l)}\left(X_{i}\right)=\lim _{\gamma \rightarrow \infty} n_{j} \frac{X_{i l}+\alpha_{l}}{n_{i}+\gamma}
$$




$$
= \begin{cases}\frac{n_{j}}{2}\left(1-\frac{L-2}{c_{l} \sum_{k=1}^{L} 1 / c_{k}}\right)=: n_{j} w_{l} & \text { for } l=1, \ldots, L, \\ 0 & \text { for } l=L+1, \ldots, r .\end{cases}
$$

For the mutual predictor $d=\left[d_{i j}\right]_{i, j=1}^{m}$ obtained in the above formula by letting $\gamma \rightarrow \infty$ the risk is (see (17))

$$
R(p, d)=\sum_{\substack{i, j=1 \\ i \neq j}}^{m} k_{i j} n_{j}^{2}\left(\sum_{l=1}^{L} q p_{l}+\sum_{l=L+1}^{r} c_{l} p_{l}+\sum_{l=1}^{L} c_{l} w_{l}^{2}\right) .
$$

The fact that the mutual predictor obtained in this way is minimax for the loss function (1) results from the following well known theorem (see [1, p. 90]) which can be adapted to the above situation.

TheOREm. If $d_{n}$ is the Bayes rule with respect to $\pi_{n}$, if $r\left(\pi_{n}, d_{n}\right) \rightarrow C$, and if $R\left(\mu, d_{0}\right) \leq C$ for all $\mu$ then the game has a value and $d_{0}$ is a minimax rule.

To define $\pi_{n}$ it is enough to put $\gamma=n$ in (12) for $\alpha_{l}$ in (18).

Then we have proved the theorem.

Theorem 2. If $A=0$ then the game defined by the statistical decision problem considered has a value and the mutual predictor $d=\left[d_{i j}\right]_{i, j=1}^{m}$ given by (19) is minimax for the loss function (1).

Up to this point we have assumed that $c_{2} \neq 0$. If only $c_{1} \neq 0$ the problem considered reduces to the minimax mutual prediction problem of a random variable $X=\left(X_{1}, \ldots, X_{m}\right)$, where $X_{j}$ 's are independent and have binomial distributions with the parameters $n_{j}, p_{1}$. The loss function is now of the form

$$
L(X, d)=\sum_{\substack{i, j=1 \\ i \neq j}}^{m} c_{1} k_{i j}\left(d_{i j}^{(1)}\left(X_{i}\right)-X_{j}\right)^{2} .
$$

In this case if $A>0$ the minimax mutual predictor $d=\left[d_{i j}\right]_{i, j=1}^{m}$ is given by

$$
d_{i j}^{(l)}\left(X_{i}\right)=n_{j} \frac{X_{i}+\gamma / 2}{n_{i}+\gamma}
$$

where the parameter $\gamma$ satisfies the equation (6), whereas when $A=0$ the minimax mutual predictor is given by the formula

$$
d_{i j}^{(1)}\left(X_{i}\right)=n_{j} / 2 .
$$

The problems of minimax estimation and prediction of binomial and multinomial random variables were considered by Hodges and Lehmann [2], Trybuła [3]-[5], Wilczyński [6] and others. 


\section{References}

[1] T. S. Ferguson, Mathematical Statistics. A Decision Theoretic Approach, Academic Press, New York, 1967.

[2] J. L. Hodges and E. L. Lehmann, Some problems in minimax point estimation, Ann. Math. Statist. 21 (1950), 182-197.

[3] S. Trybuła, Some problems of simultaneous minimax estimation, ibid. 29 (1958), 245-253.

[4] —, Minimax mutual prediction, Appl. Math. (Warsaw) 27 (2000), 437-444.

[5] - Simultaneous minimax estimation of parameters of multinomial distribution, ibid. 29 (2002), 307-311.

[6] M. Wilczyński, Minimax estimation for multinomial and multivariate hypergeometric distribution, Sankhyā Ser. A 47 (1985), 128-132.

Institute of Mathematics

Technical University of Wrocław

50-370 Wrocław, Poland

Received on 18.11.2002;

revised version on 18.4 .2003

$(1662)$ 